\title{
Genetic Reconfigurability of a Multi-Size Pixelled Antenna
}

\author{
D. Rodrigo ${ }^{(1)}$, L. Jofre ${ }^{(1)}{ }^{*}$ M. Unlu ${ }^{(2)}$, Y. Damgaci( ${ }^{(2)}$, B. A. Cetiner ${ }^{(2)}$, and J. Romeu ${ }^{(1)}$
}

(1) Signal Theory and Communications Department, Universitat Politècnica de Catalunya, Barcelona, Spain

(2) Electrical and Computer Engineering, Utah State University, Logan, Utah

\section{Reconfigurable antenna and smart systems}

Smart systems are devices which are capable of interacting with the environment by sensing it, taking decisions and adjusting their behavior accordingly. When these concepts are applied to a wireless communication system, antenna structures stand out as a key element regarding the overall performance of the smart system. In this case the smart antenna system is composed by an antenna capable of modifying its properties and a real-time synthesis algorithm controlling the antenna parameters.

Historically smart wireless systems have used antenna arrays, and adaptability has relied on the choice of the array feed values, which were determined by the synthesis algorithm [1]. A wide range of algorithms are employed for this purpose like are Capon beamformer, shrinkage based RCB and midway method [2]. The development of reconfigurable antennas allows the replacement of the array by a single reconfigurable antenna, particularly interesting under tight size constraints, and that requires a complete new approach regarding optimization techniques [3]. In this paper it is described a reconfigurable antenna suitable for smart systems as well as a real-time synthesis procedure which is based on genetic algorithms.

\section{Antenna Design}

The antenna is designed to work at $5.5 \mathrm{GHz}$ and is based in a pixelled structure, which consists in a switched grid of electrically small metallic patches [4]. The placement of a switch at several patches adjacencies allows the modification of the current distribution over the antenna and thus modifying its radiation pattern.

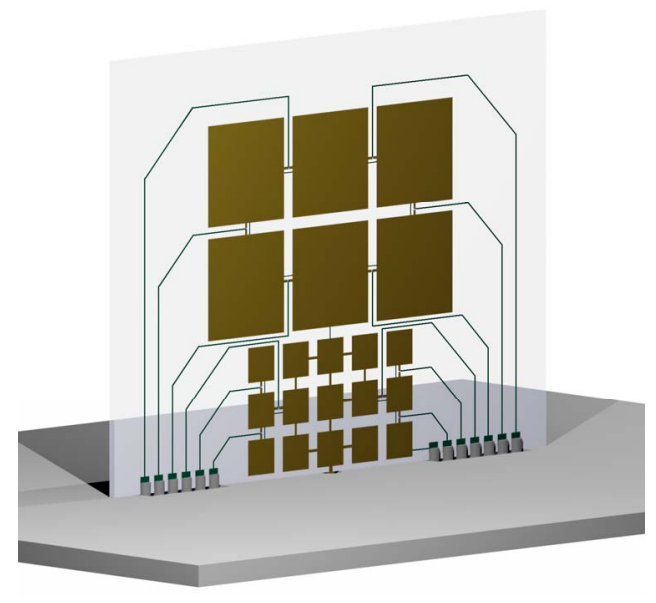

Fig. 1. Artist view of the multi-size pixelled antenna

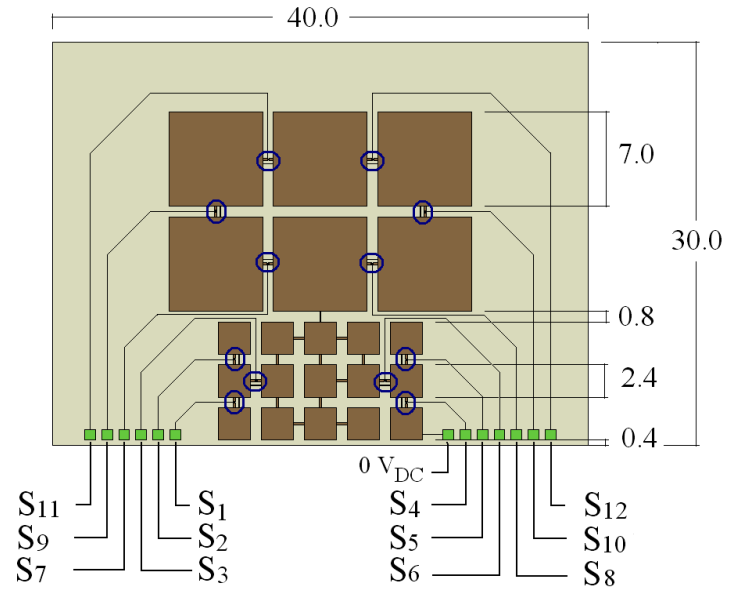

Fig. 2. Detailed sketch specifying dimensions and switch enumeration. 
This type of antenna architecture is usually based on a uniform grid of constant size patches. However, the reconfiguration capabilities of the antenna can be enhanced and the number of necessary switches reduced by using a multi-size pixel distribution. The idea behind the present geometry is to locate the different reconfiguration capabilities on different antenna regions. The lower region which is near to the $\mathrm{RF}$ input port is responsible of the antenna matching, while the upper region allows the modification of the radiation pattern. In order to minimize the influence of the upper part over the input impedance, this region is left as a parasitic area. This isolation of functionalities allows an independent optimization of the pixel size for each region. Since the input impedance is very sensitive to any dimensional change a fine grid of patches has been designed for the lower region. On the other hand, to modify the antenna radiation pattern strong modifications over the current distribution are required, which makes more convenient the use of larger patches in its corresponding region.

The position of the switches has been determined by optimizing a fully switched model (a switch between in pair of adjacent patches) under different conditions and replacing the switches with the less variable status by gaps or wires depending on the predominant status. In order to reduce the complexity of the design, this criterion has been made compatible with the possibility of placing all the switches bias lines in the same layer of the substrate, leading to a total number of necessary switches equal to 12 .

\section{Genetic Synthesis Procedure}

In order to take advantage of the antenna reconfigurability an optimization algorithm is needed. This optimization algorithm determines the status of each one the switches in order to synthesize a certain radiation pattern. The criteria followed for this purpose is the maximization of the antenna gain in a specific direction, which means that the synthesis procedure is running an joint optimization of both the radiation pattern, in order to have a maximum in the desired direction, and also the reflection coefficient, in order to reduce the losses due to mismatch. The situation may be formulated as the following discrete optimization problem

$$
\max _{s_{i} \in\{\mathrm{ON}, \mathrm{OFF}\}, i=1 . .12} D\left(\theta_{0}, \varphi_{0}\right)-10 \log \left(1-\left|s_{11}\right|^{2}\right)
$$

where $D$ is the directivity, and $\theta_{0}, \varphi_{0}$ represent the direction of interest.

Suitable optimization algorithms in this case are those capable of dealing with a discrete space of parameters, such are genetic algorithms [5], neural networks [6] and particle swarm optimization [7]. In this case a genetic algorithm has been employed due to its good performance regarding convergence speed and avoidance of local maximums when applied to electromagnetic problems.

The implementation of the genetic procedure has been coded in Matlab, controlling also the launching of the electromagnetic simulations required to the compute the fitness of each configuration. These EM simulations have been performed using a full wave finite element method with models that include the high-resistive bias lines and the exact geometry of the switches. In order to obtain good rates of convergence the parameters of the genetic algorithm must be chosen properly. In this case, a value of 15 for the population of each generation and a $5 \%$ of individual mutation probability lead to convergence in approximately 15 iterations. The choice of these two parameters is especially important in a real-time optimization situation. Low values of population with high mutation probabilities lead to fast-response smart antennas, suitable for highly changing environments where the channel presents a small coherence time. On the other hand, high values of population and low mutation probabilities reduce the adaptation speed but lead to more stable configurations closer to optimal solution, what made them appropriate for slow changing environments. 


\section{Simulations and Measurements}

The genetic synthesis algorithm has been applied for maximization of the antenna gain at 4 different direction of arrival $\left(-60^{\circ},-30^{\circ}, 30^{\circ}\right.$ and $\left.60^{\circ}\right)$. The resulting switch configurations after 15 iterations are presented in Table I, where 0 and 1 represent an ON and OFF status respectively and the switch enumeration corresponds to Fig. 2.

In Fig. 3 and Fig. 4 is represented respectively the radiation pattern and reflection coefficient of each configuration. It can be observed that the maximum of the radiation patterns is pointing to the desired direction in each case, with directivity between $6 \mathrm{~dB}$ and $8 \mathrm{~dB}$, which is a high value taking into account that the overall size of the antenna is around half-wavelength. At the same time, the different configurations present resonances close to the design frequency $(5.5 \mathrm{GHz})$ with values of return losses below $10 \mathrm{~dB}$.

Table I: Switch Configurations

\begin{tabular}{cccccccccccccc}
\hline & & $\mathrm{S}_{1}$ & $\mathrm{~S}_{2}$ & $\mathrm{~S}_{3}$ & $\mathrm{~S}_{4}$ & $\mathrm{~S}_{5}$ & $\mathrm{~S}_{6}$ & $\mathrm{~S}_{7}$ & $\mathrm{~S}_{8}$ & $\mathrm{~S}_{9}$ & $\mathrm{~S}_{10}$ & $\mathrm{~S}_{11}$ & $\mathrm{~S}_{12}$ \\
\hline DOA & $-60^{\circ}$ & 0 & 0 & 1 & 0 & 0 & 1 & 0 & 0 & 1 & 0 & 0 & 1 \\
DOA & $-30^{\circ}$ & 1 & 0 & 0 & 0 & 1 & 1 & 1 & 0 & 1 & 1 & 0 & 1 \\
DOA & $30^{\circ}$ & 0 & 1 & 0 & 1 & 0 & 1 & 0 & 0 & 1 & 1 & 1 & 0 \\
DOA & $60^{\circ}$ & 0 & 1 & 0 & 0 & 1 & 1 & 0 & 1 & 1 & 1 & 1 & 0 \\
\hline
\end{tabular}

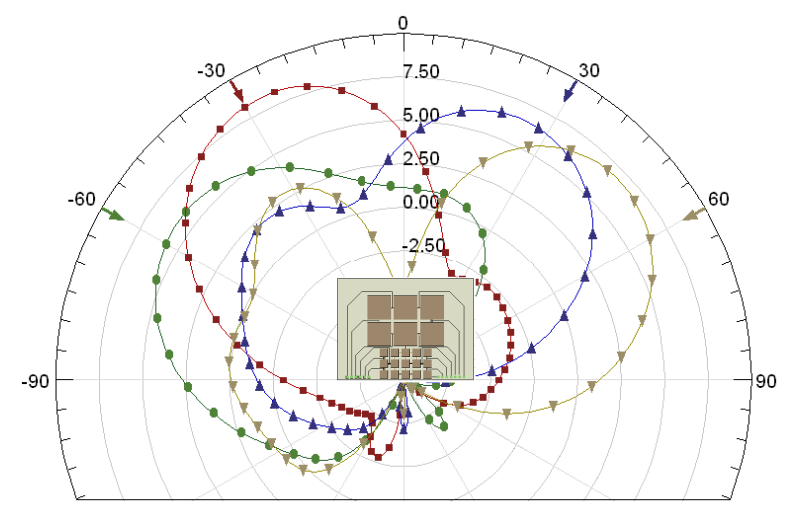

Fig. 3, Radiation pattern of the 4 optimized configurations

To experimentally validate these results, a simpler switched prototype (Fig. 5) capable of steering the main beam towards two different directions $\left(-30^{\circ}\right.$ and $30^{\circ}$ ) has been manufactured. Since only 2 configurations have been taken into account, the number of necessary switches has been reduced to 4 .

The measures of the radiation pattern and the reflection coefficient are presented in Fig. 6 and Fig. 7 respectively. It can be observed a good agreement between simulations and measures, with radiation patterns steered to the desired direction while maintaining a good matching.

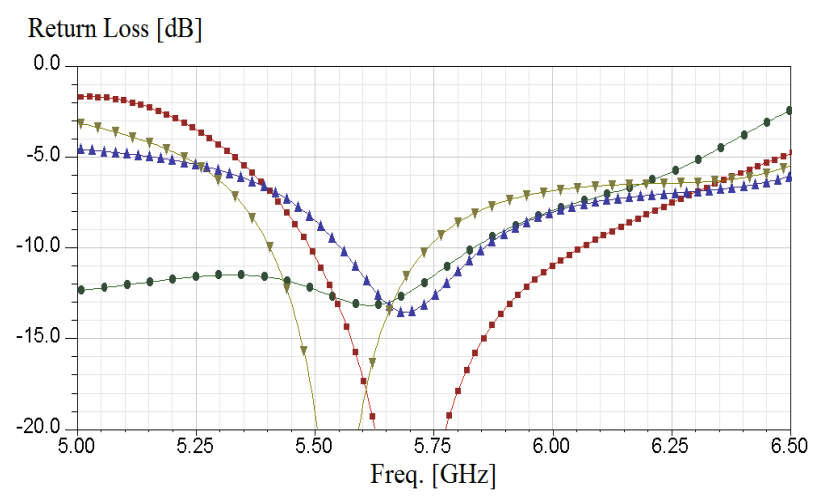

Fig. 4, Reflection coefficient of the 4 optimized configurations

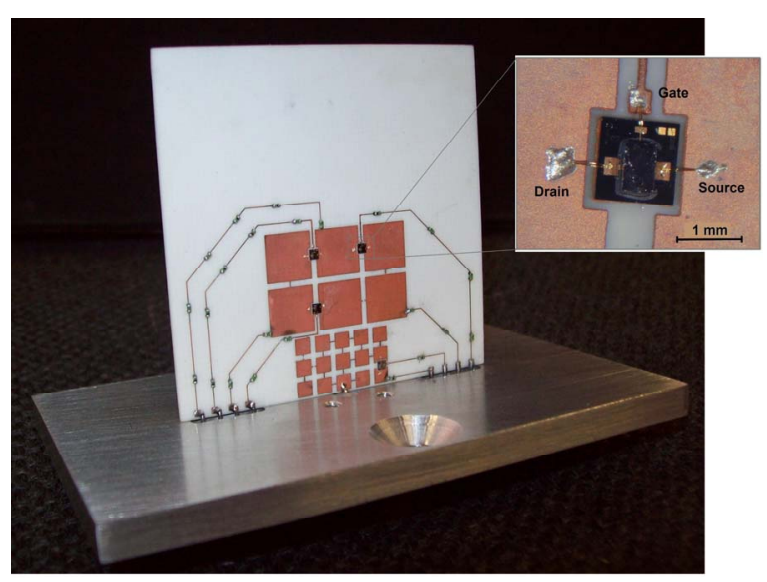

Fig.5, Reconfigurable pixelled monopole prototype 


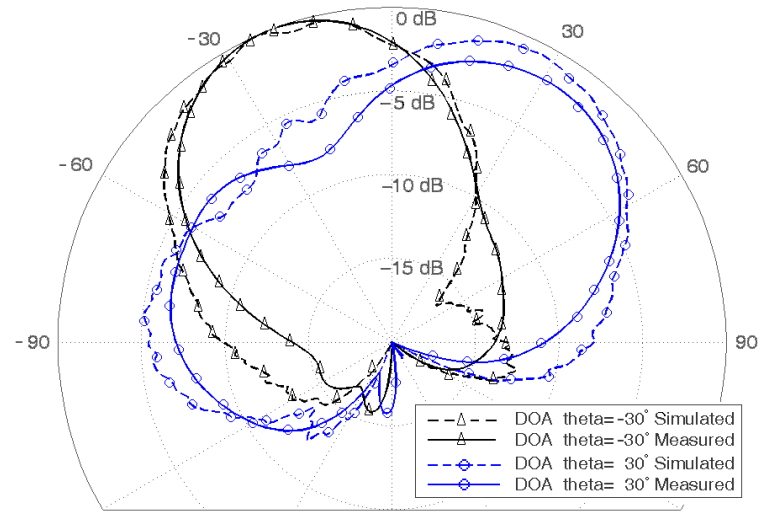

Fig. 6, Radiation pattern of the 2 simulated (dashed) and measured (solid) configurations

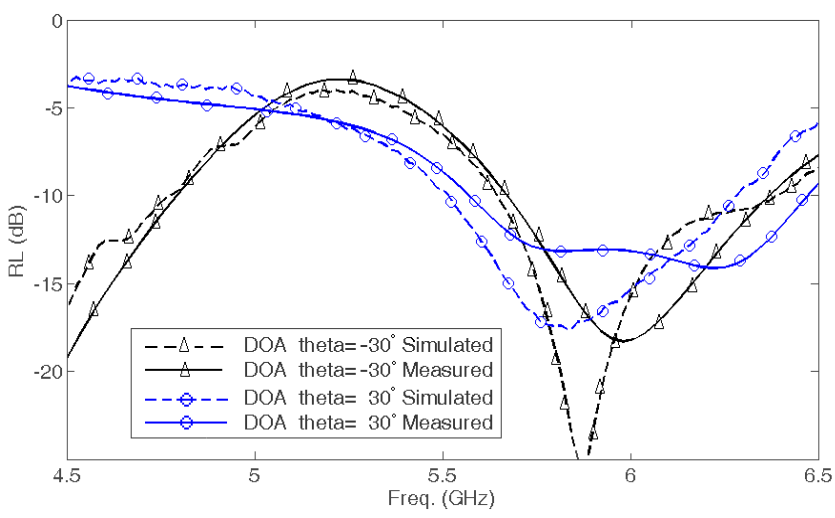

Fig. 7, Reflection coefficient of the 2 simulated (dashed) and measured (solid) configurations

\section{Conclusions}

A smart antenna system has been designed combining reconfigurable antenna concepts with genetic optimization algorithms.

The employment of different sized patches in the antenna geometry has allowed the use of a simpler structure with a low number of switches while maintaining a good reconfiguration capability.

The combination of the previous antenna with genetic synthesis algorithms have made up a smart antenna system capable of steering the radiation pattern over an angular sector of $120^{\circ}$.

A simpler prototype has been manufactured and measured, presenting a good agreement with simulated results.

\section{References:}

[1] Bellofiore, S.; Balanis, C.A.; Foutz, J.; Spanias, A.S. "Smart-antenna systems for mobile communication networks. Part 1. Overview and antenna design," Antennas and Propagation Magazine, IEEE, vol.44, no.3, pp. 145- 154, Jun 2002

[2] Gotsis, K.A.; Siakavara, K.; Sahalos, J.N.; , "On the Direction of Arrival (DoA) Estimation for a Switched-Beam Antenna System Using Neural Networks," Antennas and Propagation, IEEE Transactions on, vol.57, no.5, pp.1399-1411, May 2009

[3] A. Grau, J. Romeu, L. Jofre, and F. De Flaviis. "A software defined MEMS reconfigurable pixelantenna for narrowband MIMO systems," In Adaptive Hardware and Systems, 2008. AHS '08. NASA/ESA Conference on, pp: 141-146, June 2008

[4] Pringle, L.N. et al. , "A reconfigurable aperture antenna based on switched links between electrically small metallic patches," Antennas and Propagation, IEEE Transactions on , vol.52, no.6, pp. 14341445, June 2004

[5] Yahya Rahmat-Samii and Eric Michielssen, editors. "Electromagnetic Optimization by Genetic Algorithms". John Wiley \& Sons, Inc., New York, NY, USA, 1999.

[6] Gotsis, K.A.; Siakavara, K.; Sahalos, J.N.; , "On the Direction of Arrival (DoA) Estimation for a Switched-Beam Antenna System Using Neural Networks," Antennas and Propagation, IEEE Transactions on, vol.57, no.5, pp.1399-1411, May 2009

[7] Gies, D.; Rahmat-Samii, Y.; , "Reconfigurable array design using parallel particle swarm optimization," Antennas and Propagation Society International Symposium, 2003. IEEE , vol.1, no., pp. 177- 180 vol.1, 22-27 June 2003 